\title{
Optical Bistability in a SOA/DFB-LD Feedback Scheme
}

\author{
W. D’Oosterlinck, G. Morthier, R. Baets \\ Department of Information Technology, Ghent University - IMEC, Sint-Pietersnieuwstraat 41, B-9000 Gent, Belgium \\ Tel.: +3292648930. ,E-mail:wdooster@intec.ugent.be \\ T. Erneux \\ Optique Nonlinéaire Théorique,Université Libre de Bruxelles, Campus Plaine CP 231, B-1050 Bruxelles, Belgium
}

\begin{abstract}
Optical bistability is investigated using a DFB laser diode connected with a SOA. Broadband operation and tunability of the domain of bistable responses is shown. The device can readily serve as an all-optical flip-flop.

(C)2006 Optical Society of America

OCIS codes: (250.0250) Optoelectronics; (250.5980) Semiconductor Optical Amplifiers; (250.5300) Photonic Integrated Circuits
\end{abstract}

\section{Introduction}

All-optical packet switching is gaining a lot of interest mainly due to the huge growth of Internet traffic. One of the key building blocks to come to such networks is the optical flip-flop [1]. These optical flip-flops usually rely on some sort of optical bistable response and a number of solutions have already been proposed in the literature [2-3]. In this paper we show that optical bistability can be obtained from a travelling wave SOA connected to a DFB laser diode. The same device has been proposed as an optical decision circuit [4] but here we show that it exhibits bistable behaviour under the right operation conditions. This device offers several advantages over existing bistable devices or flip-flops, such as the broad wavelength range for the input signals (which makes it possible to use the same device for different wavelength channels), the simple configuration and also the easy tunability of the domain of bistable responses. We also point out that the bistability reported here is not the narrowband dispersive bistability observed in a DFB amplifier. The DFB is biased above threshold in our scheme and the input wavelength is far from the lasing wavelength.

\section{Device description}

The device used in our experiments is a DFB laser diode integrated on a single chip with a SOA and a coupler, as can be seen schematically in Fig.1. Through the coupler a part of the output power of the SOA is coupled into the laser diode and at the same time a part of the laser power is coupled into the SOA. In this way a feedback scheme between laser diode and SOA is obtained.

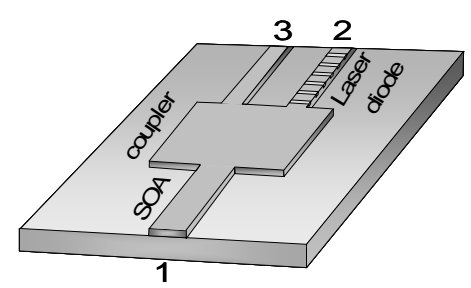

Fig.1. Schematic representation of the integrated SOA/DFB laser diode feedback scheme

In the actual device the coupler is a 1X4-coupler that connects a SOA with 4 different laser diodes [5], each operating at a different wavelength, of which only one is used.

During the measurements light from a tuneable laser was injected into the SOA using a lensed fiber (at port 1). For practical reasons the output power was obtained at the same side and separated from the input power by a circulator, however, we could also use the signal obtained at ports 2 and 3 (respectively after the laser diode and a waveguide). As output power both the reflected input signal (due to reflections at the back facet of the DFB laser diode) and the laser power can be used after separating them from each other using a tuneable optical filter. The laser diode is operated well above threshold.

The device is essentially relying on the interplay between the amplified input power and the laser power in both the SOA and the laser diode. For a certain input power range, there are two stable states. In one state the SOA gives a high amplification, with much power injected into the laser and the laser switches off. The absence of laser light 
injection into the SOA then guarantees the high amplification. In another state, the SOA is highly saturated and less power is injected into the laser. The laser is not switched off and injects sufficient light into the SOA to saturate it. A theoretical analysis, reported elsewhere [6], indicates that bistability occurs only for sufficiently high amplifier currents, or sufficiently low laser diode currents.

\section{Experimental results}

For the experiments light at a certain wavelength was injected into the device and the laser signal, at a wavelength of $1540 \mathrm{~nm}$, was used as output power of the device. The drive current for the laser diode was $87 \mathrm{~mA}$.
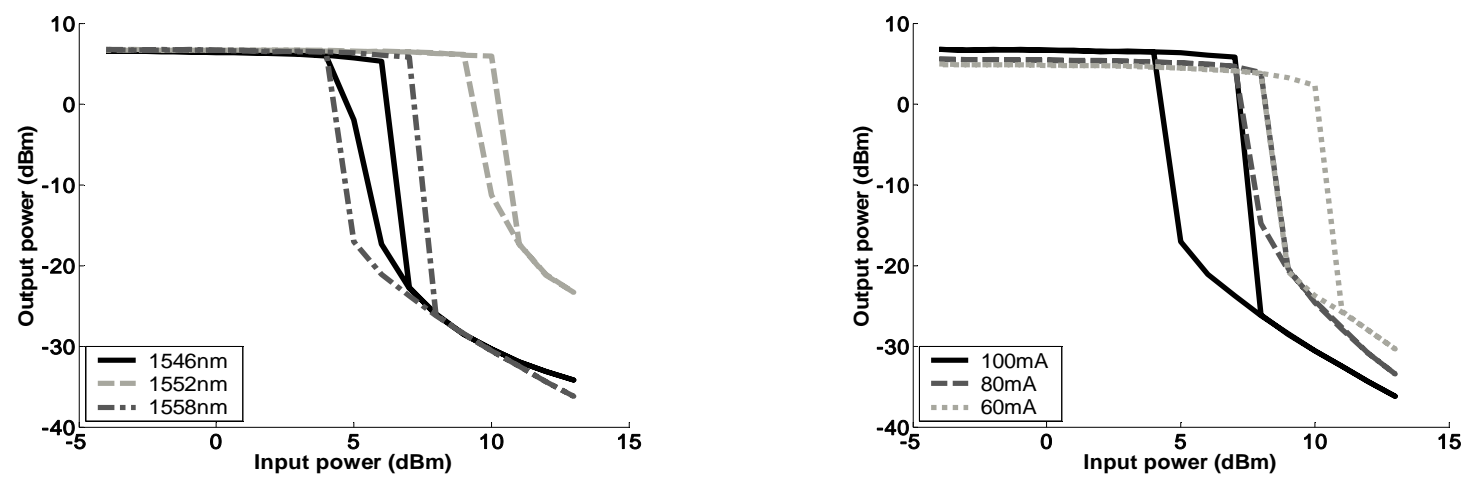

Fig. 2: Mesaured optical output vs. input power relation for different wavelengths (left) and different drive current for the SOA (right).

In Fig. 2 it is shown that with constant drive currents for SOA $(100 \mathrm{~mA})$ and laser diode, bistability can be obtained over a broad wavelength range, but with a change of the domain of bistability along the input power axis. It is also shown that by varying the drive current of the SOA, for a fixed input signal wavelength of $1555 \mathrm{~nm}$, this domain of bistability can be shifted as well. In both cases the height and width of the bistability vary respectively between 20 to $25 \mathrm{~dB}$ and 1 to $3 \mathrm{~dB}$. The same behaviour can be observed in the signal output power, but with an upward step. When used as an optical flip-flop, the device of Fig. 1 can in principle be set by an optical pulse at port 1 and reset by a pulse at port 3 or vice versa. The switching energy will be determined in the near future. The width of the bistability is however relatively small, suggesting that a small switching energy can be expected.

\section{Conclusion}

We have shown that optical bistability can be obtained using a scheme consisting of a DFB laser diode and a SOA. We show that both the height and the position of the bistable domain can be changed by adjusting just one of the drive currents. The broadband operation and the simple structure make it a very robust and flexible device.

\section{References}

[1] H.J.S. Dorren, M.T. Hill, Y. Liu, N. Calabretta, A. Srivatsa, F. M. Huijskens, H. de Waardt, G. D. Khoe, “Optical Packet Switching and Buffering by Using All-Optical Signal Processing Methods”, Journal of Lightwave Technology, vol. 21, jan 2003, pp. 2-12.

[2] E. Tangdiongga, X.L. Wang, Z.G. Li, Y. Liu, D. Lenstra, H. Dorren, “Optical flip-flop based on two-coupled mode-locked ring lasers”, IEEE Photon. Technol. Lett., vol 17, jan 2005, pp. 208-210.

[3] H. Kawaguchi, “Bistable laser diodes and their applications: state of the art”, IEEE J. Sel. Topics Quantum Electron., vol. 3, oct 1997, pp $1254-1270$.

[4] W. D’Oosterlinck,G.Morthier,R. Baets, M.K. Smit, “ Very steep optical thresholding characteristic using a DFB laser diode and an SOA in an optical feedback scheme”, IEEE Photon. Technol. Lett., vol. 17, mar 2005, pp.642-644.

[5] K. Yashiki, K. Sato, T. Morimoto, S. Sudo, K. Naniwae, S. Ae, K. Shiba, N. Suzuki, T. Sasaki and K. Kudo, "Wavelength-Selectable Light Sources Fabricated Using Advanced Microarray-Selective Epitaxy," IEEE Photon. Technol. Lett., vol. 16, pp. 1619-1621, 2004.

[6] W. D’Oosterlinck, T. Erneux, G. Morthier, to be published.

\section{Acknowledgments}

The work is supported by the InterUniversity Attraction Pole program of the Belgian government and the Fonds National de La recherché Scientifique (Belgium). Wouter D'Oosterlinck acknowledges the Flemish IWT for financial support and M.K. Smit. The authors would like to thank K. Kudo, K. Yashiki, K. Sato, and J. De Merlier from NEC Corporation for lending wavelength-selectable light sources. 


\section{CLEO

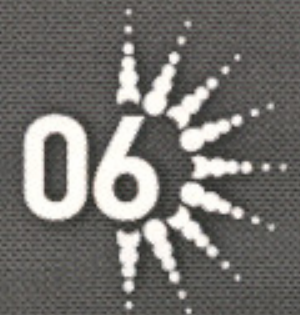

Fis otonic

- Pplications

Systems

is echnologies"

Conference on Lasers and Electro-0ptics Quantum Electronics and Laser Science Conference Conference on Photonic Applications, Systems and Technologies

\section{Technical Digest CD-ROM}

ISBN: 1-55752-813-6

Long Beach Convention Center Long Beach, California, USA

CLEO/QELS Conference: May 21-26, 2006 PhAST Conference: May 22-25, 2006

www.cleoconference.org • www.phastconference.org
Table of Contents

CLEO/QELS and PhAST

Program Committees

Agenda of Sessions

Abstracts

Key to Authors and Presiders

Citation Information

Search this CD-ROM

CD-ROM Help

(92006 